\title{
Duplication of the Inferior Vena Cava, Azygos Continuation and Retro Aortic Renal Vein: Fortuitous Discovery
}

\author{
Géraud Léra Akpo ${ }^{1}$, Ndeye Bigué Mar ${ }^{12^{*}}$, Soukeyna Abouabdillah', Nfaly Badji1, Hamidou Deme ${ }^{1}$, \\ Youssoupha Kasse ${ }^{1}$, Ibrahima Niang ${ }^{3}$, Mamadou Lamine Gueye ${ }^{3}$, Marcel Mbarga ${ }^{1}$, \\ Ibrahima Faye1, Abdoulaye Dione Diop4, Assane Ndiaye5, Sokhna Ba Diop³, El Hadji Niang1 \\ ${ }^{1}$ Service de Radiologie, Imagerie Médicale de l'hôpital Ariste Le Dantec, Dakar, Sénégal \\ ${ }^{2}$ Laboratoire d'Anatomie UFR des Sciences de la Santé Université Iba Der Thiam de Thiès, Thiès, Sénégal \\ ${ }^{3}$ Service de Radiologie, Imagerie Médicale de l'hôpital Fann, Dakar, Sénégal \\ ${ }^{4}$ Service de Radiologie, Imagerie Médicale de l'hôpital Dalal Jam, Dakar, Sénégal \\ ${ }^{5}$ Laboratoire d'Anatomie UFR des Sciences de la Santé Université Assane SECK de Ziguinchor, Ziguinchor, Sénégal \\ Email: ^biguemarmbaye@yahoo.fr
}

How to cite this paper: Akpo, G.L., Mar, N.B., Abouabdillah, S., Badji, N., Deme, H., Kasse, Y., Niang, I., Gueye, M.L., Mbarga, M., Faye, I., Diop, A.D., Ndiaye, A., Diop, S.B. and Niang, E.H. (2021) Duplication of the Inferior Vena Cava, Azygos Continuation and Retro Aortic Renal Vein: Fortuitous Discovery. Forensic Medicine and Anatomy Research, 9, 31-40. https://doi.org/10.4236/fmar.2021.93004

Received: April 26, 2021

Accepted: June 19, 2021

Published: June 22, 2021

Copyright ( 2021 by author(s) and Scientific Research Publishing Inc. This work is licensed under the Creative Commons Attribution International License (CC BY 4.0).

http://creativecommons.org/licenses/by/4.0/

\begin{abstract}
We present a rare case associating inferior vena cava's (IVC) complex variations. Anatomical, embryological and clinical aspects are discussed. Duplication's discovery was fortuitous during an abdominal ultrasound and was confirmed by the CT scan. The latter also highlighted azygos continuation, retro aortic renal vein and an iliac venous anomaly. These variants are important to know in order to be able to differentiate them from lymphadenopathy, para-vertebral masses, and aortic pathologies. It is necessary to report them before any retroperitoneal surgery or interventional vascular procedure.
\end{abstract}

\section{Keywords}

Duplication, IVC, Continuation, Azygos, Tomodensitometry

\section{Introduction}

The prevalence of IBV developmental abnormalities in the general population ranges from $0.07 \%$ to $8.7 \%$ [1]. They are usually discovered during autopsies, but are increasingly found with the latest imaging techniques [2]. These abnormalities are usually clinically silent [3]. They provide thrombosis and their knowledge helps to reduce or eliminate the risk of severe bleeding during retroperitoneal surgery [2]. We report a case combining a duplication of the inferior vena cava (VCI), an azygos continuation, a retroaortic renal vein and an anatomical 
variant of the iliac veins. The discovery was incidental during an abdominal ultrasound and was confirmed at CT. The presence of such a combination is rare in the literature.

\section{Observation}

It is a 69-year-old patient, hypertensive, carrier of the antigen HBs (marker viral hepatitis B), addressed to the radiology department of the hospital Aristide Le Dantec for a recurrent diffuse abdominal pain with alteration of the general state. No surgical history was found.

The clinical examination found a soft abdomen, without palpable mass. The cardiac auscultation did not find overheard noises and the vascular examination found peripheral vascular pulses perceptible on the different arterial axes.

The biological assessment revealed the presence of Ag HBs with the absence of Ac anti HBs. Creatinine was $12 \mathrm{mg} / \mathrm{l}$, the blood count was negative for anemia and thrombocytopenia. Liver function showed no hepatic cytolysis or cholestasis.

Abdominal-pelvic ultrasound with ultrasound (SUPERSONIC Magic) and $3.5 \mathrm{MHz}$ low-frequency probe coupled to B-Mode and Doppler.

This examination revealed the presence of a left lateral aortic structure, oval in the axial plane, tubular in the frontal plane, with a venous Doppler signal (Figure 1 and Figure 2). This venous structure originates from the left external iliac vein, passes through the left aortic lateral passage, and flows into the left renal vein (Figure 3). The left internal iliac vein is not visible. The IBV, located to the right of the abdominal aorta, is formed by the confluence of the right internal and external iliac veins. The left renal vein flows behind the aorta and into the IBV (Figure 4).

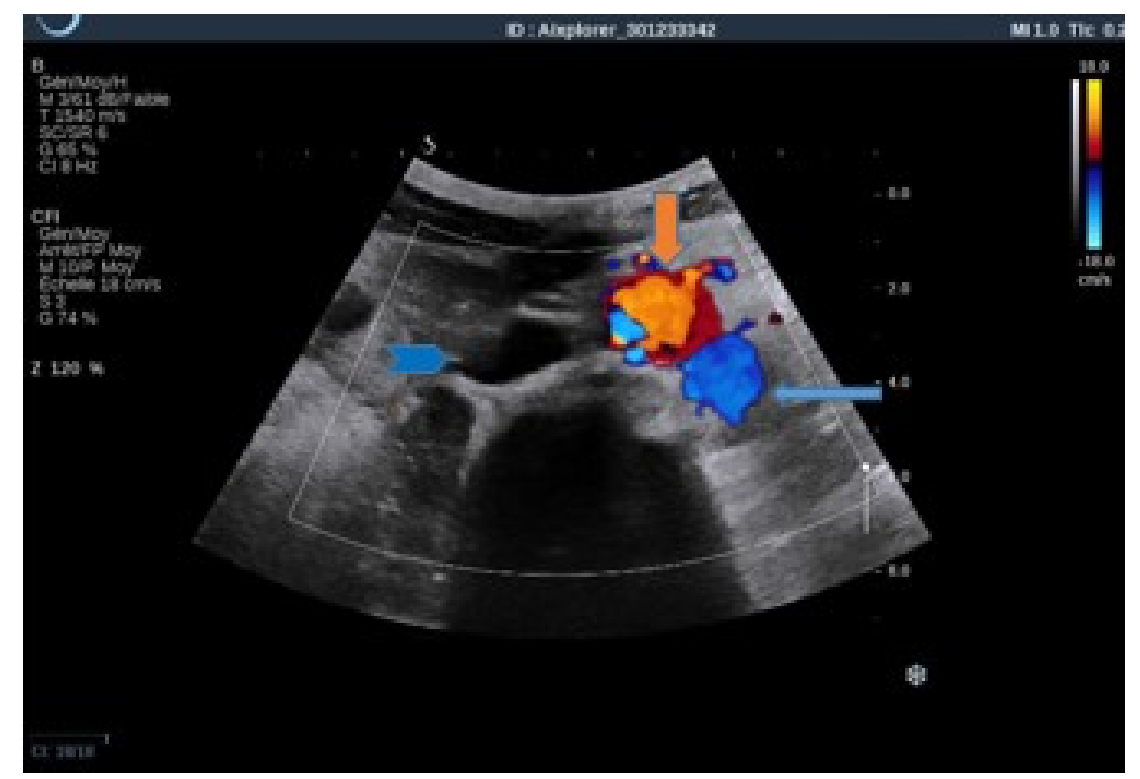

Figure 1. Cross-sectional ultrasound section of the umbilical region showing the abdominal aorta (orange arrow), ICV (arrowhead) and left aortic lateral vascular structure. 


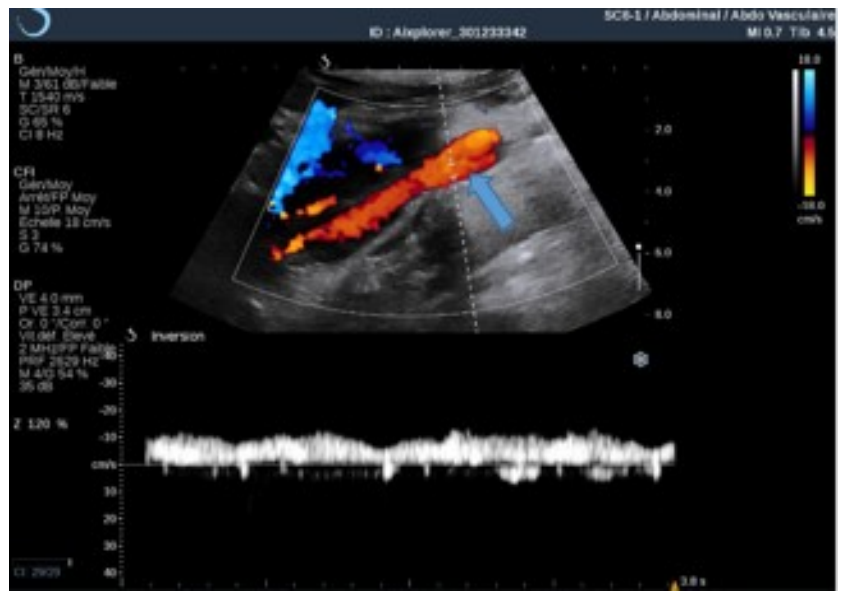

Figure 2. Longitudinal ultrasound image of the umbilical region showing flow in the left vena cava (blue arrow).

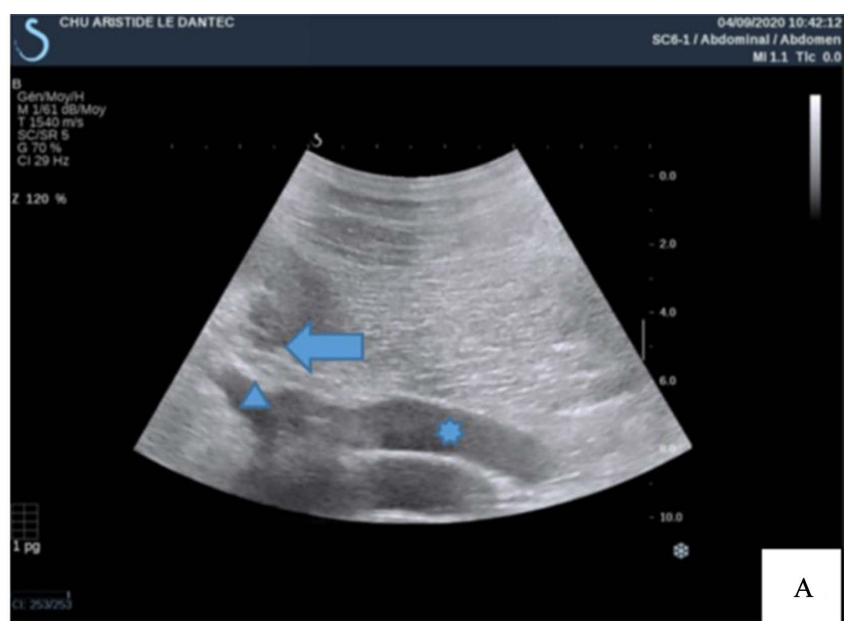

Figure 3. Axial angular ultrasound slice in right lateral decubitus in B-mode passing through the left renal hilum, showing the left (star) IFV draining into the left renal vein.

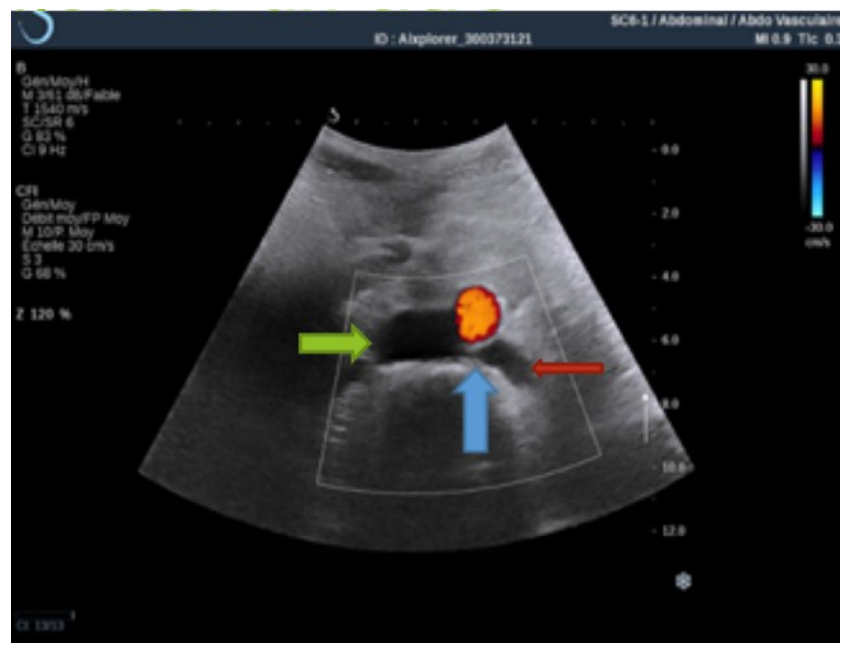

Figure 4. Cross-section ultrasound of the epigastric region showing the retro-aortic passage (blue arrow) of the left renal vein (red arrow) before jumping into the "right" ICV (green arrow). 
Liver analysis showed drainage of the hepatic veins directly to the heart mass, with no visualization of retrohepatic IBV.

The liver was not dysmorphic, homogeneous apart from some biliary cysts.

These aspects made us evoke a double inferior vena cava.

Ultrasound exploration of abdominal vascular structures was partially hampered by digestive gas interpositions.

In addition, there was a single millimetre bilateral inferior polar renal cyst and moderate diffuse aerocolic.

Before these ultrasound images, two diagnostic hypotheses were discussed: a dilated gonadal vein or thrombosis of the hepatic retro vena cava. A complementary scanner (SIEMENS ${ }^{\circledR}$ SOMATOM definition AS 64 strips) with injection of iodized contrast agent at arterial and venous times was performed. The exam protocol included a chest-abdominal-pelvic scan with a $90 \mathrm{ml}$ injection of iodized contrast agent at $350 \mathrm{mg} / \mathrm{ml}\left(\mathrm{OMNIPAQUE}^{\oplus}\right)$ at arterial and portal times. Reconstructions, sagittal, coronal MIP and VRT were performed.

The CT had confirmed a duplication of the inferior vena cava, with a left ICV extending the left external iliac vein, moving up the left side of the abdominal aorta (Figure 5) to the left renal vein (Figure 6). The latter passes behind the aorta and ends by jumping into the right IFV (Figure 7). The left renal vein is increased by a $13 \mathrm{~mm}$ caliber.

The IBV originates from the confluence of the right internal and external iliac veins, then follows a classic path; receiving the right and left renal veins. It stops abruptly before entering the liver. The left internal iliac vein flows into the right ICV.

The dilated azygo vein then takes over by draining all the blood from the IBV and passing through the posterior infra mediastinal space through the right pillar of the diaphragm (Figure 7 and Figure 8). It rises along the right side of the thoracic spine and flows into the VCS next to T4.

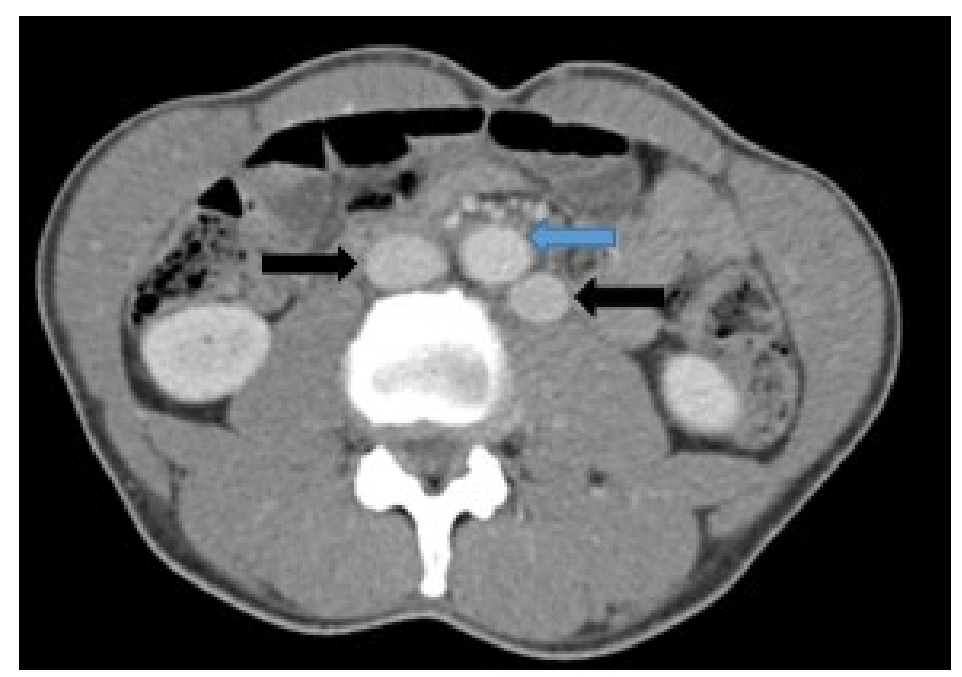

Figure 5. Axial scan image after contrast injection showing the two IVCs (black arrows) on either side of the abdominal aorta (blue arrow). 


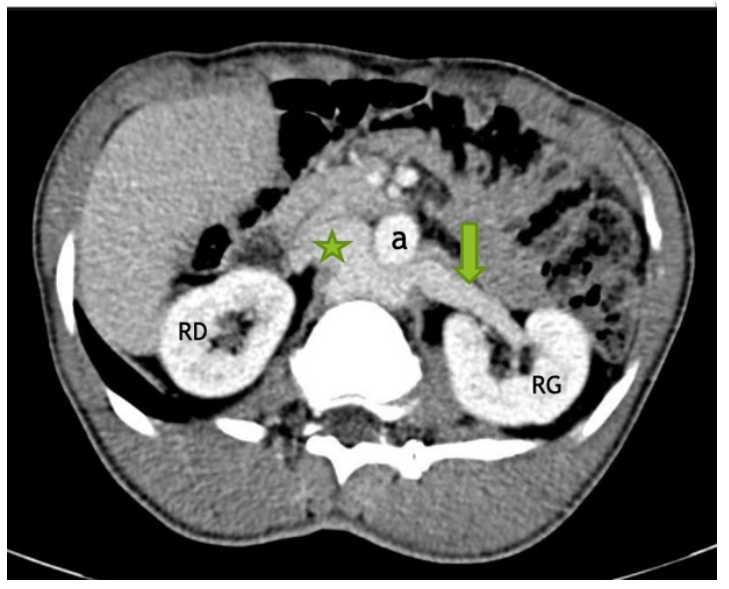

Figure 6. Volume reconstruction (MPR) scannographic image, showing the duplication of the inferior vena cava, azygos continuation, and left renal vein.

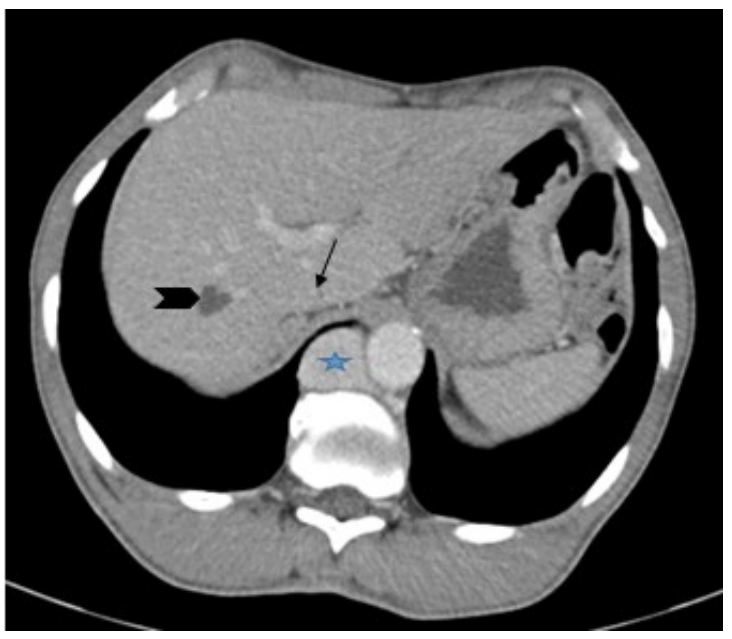

Figure 7. Axial CT scan showing the absence of the retrohepatic portion of the IVC (arrow) and the existence of a dilated posterior infra mediatisnal vein corresponding to the azygos vein (star). Segment 8 hepatic cyst (arrowhead).

The azygos vein is increased in caliber $(27 \mathrm{~mm})$ over its entire path, making with the arch of the aorta an aspect in double butt.

The right inferior vena cava is larger $(27 \mathrm{~mm})$ than its left counterpart (17.5 $\mathrm{mm})$.

Since the retro-hepatic portion of the IFV is not present, the hepatic veins in the modal position are drained directly into a small segment of the IFV before being thrown into the right atrium of the heart.

Thus, we did not find any organic cause for the abdominal pain mentioned at admission.

The patient and his treating physician were informed of these findings. In the face of functional pain, only symptomatic treatment with antispasmodic and activated charcoal has been instituted. We recommended a regular hepatic control ultrasound to monitor for cirrhotic changes in the liver. No surgical treatment was indicated. 


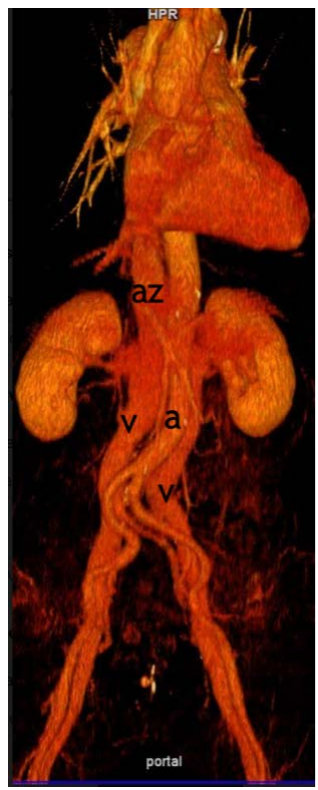

Figure 8. CT image in volume reconstruction (MPR), showing the duplication of the inferior vena cava, the azygos continuation, and the left renal vein. a: aorta; az: azygos vein; v: inferiorvena cava.

\section{Discussion}

The prevalences of the duplication of the inferior vena cava, azygos continuation and retroaortic renal vein are respectively $0.2 \%-3 \% ; 0.6 \%$ and $2.1 \%$. The presence of such a combination of variations is very rare [1] [4]. In this observation the patient presents a complex variation of the IBV including: the hepatic, renal, sub-renal and ilio-cave segment.

Remember that the normal IBV is composed of 6 segments: hepatocardiac, hepatic, suprarenal, renal, sub-renal and ilio-cave.

The evolution towards the definitive cave system is made from three pairs of primitive veins: the posterior cardinal veins, the sub-cardinal veins and the supra-cardinal veins. These systems will develop and regress at different times with some temporal overlap, establishing numerous anastomoses between them [5] [6].

According to Bass, the major abnormalities are double IBV (with a prevalence of $0.2 \%-3 \%)$, left IBV $(0.2 \%-0.5 \%)$, left renal vein retro aortic $(2.1 \%)$, left renal vein circum aortic (8.7\%) and the absence of the liver segment of IBV with azygos prolongation of IBV (0.6\%) [3] [7].

The duplication of the IVV is characterized by the presence of a second inferior vena cava sub renal. It results from the persistence of the right and left supra-cardinal veins while the left supra-cardinal vein should involuer.

The left ICV can be located to the left of the abdominal aorta as in our observation or more rarely to the right of the same side as the right ICV [8].

In our patient, there was no communication between the two cellars veins but in about $70 \%$ of reported cases, we find inter iliac anastomosis. This is presented as a vein draining blood from a IVV to the contralateral one [9]. 
Taking into account the size of the double ICV and the pre-aortic trunk, a morphological classification of this anomaly was proposed [4].

Type I of duplication refers to a bilateral and symmetrical ICV of the same caliber as the pre aortic trunk. In Type II, the two left and right IVCs are symmetrical but their calibres are smaller than those of the pre aortic trunk. For Type III, the left IBV caliber is smaller than the right IBV and the pre aortic trunk caliber.

In our patient, the caliber measured on the scanner was $11 \mathrm{~mm}$ for the left VCI and $19 \mathrm{~mm}$ for the right VCI; suggesting a Type III duplication.

Type III duplication would be the expression of a partial regression of the left supra cardinal vein [4].

The discovery of this malformation is often fortuitous as in our patient. Most cases of duplicated IBV are clinically silent, incidentally identified during imaging, surgery or autopsy [3] [4] [10].

The clinical impact of this anomaly remains minor. However, this ilio-cave malformation can lead to several types of clinical manifestations, such as deep venous thrombosis (DVT), extrinsic urinary obstruction, nutcracker syndrome and pelvic congestion. Numerous case studies of idiopathic DVT in children have revealed an abnormality of IBD [6] [11]. Deep venous thrombosis (DVT) of the lower limbs is found in $6.5 \%$ of cases with inferior vena cava duplicity [5]. Duplication of the inferior vena cava would cause $0.5 \%$ unexplained macroscopic hematuria and does not counter-indicate kidney transplantation [12] [13]. It can be confused with lymphadenopathy or lymphomatous flow [2] [4].

Recognition of IBV duplication is relevant in retroperitoneal surgery (especially thora-phreno-laparotomy) given the risk of massive per-operative hemorrhage in the event of unfamiliarity or vascular interventions, to avoid recurrent pulmonary embolism after the installation of a cave filter [1] [2] [3] [4].

Azygos continuation of the VCI also called agenesis of the hepatic segment of the VCI with azygos continuation is a congenital abnormality in which the retrohepatic portion of the inferior vena cava is absent. Venous drainage of the suprarenal segment is done via the azygos vein. The hepatic veins are drained directly into the right atrium through the inter-hepatocardiac segment of the IBV as in our patient [2] [3] [13] [14].

The embryological basis is the lack of formation of the right sub-cardinal anastomosis. This rare abnormality has a prevalence of $0.6 \%$ in patients with congenital heart disease, but less than $0.1 \%$ in the general population [13].

Clinically, asymptomatically, it is often incidental. Azygos continuation is found in $5 \%$ of patients under 30 years of age with unexplained deep vein thrombosis [14].

This congenital abnormality may be isolated, but is often part of a more complex syndrome that includes heart defects, situs inversus or polysplene syndrome [1] [10] [12]. In our observation, this anomaly was isolated. It can be suspected before a mediastinal enlargement to the chest radiography evoking in addition a right paratracheal mass or a para-vertebral polyadenopathy [2] [3] [13]. It can 
also mimic aortic pathology, because these dilated vessels are parallel to the descending thoracic aorta, and can be confused with aortic pathology (dissection, aneurysm or rupture) during trans-esophageal echocardiography [1].

In young patients, IVV abnormalities should be systematically investigated for recurrent and idiopathic DVT [11] [15].

It is important to know the anatomy of this variant before planning a cardiac bypass, cardiac catheterization, azygo ligature during a thoracotomy or porto-cave decompression surgery [1] [2] [16].

The retroaortic left renal vein results from the regression of supra-sub-cardinal anterior anastomosis and the persistence of posterior supra-sub-cardinal anastomosis, so that a single renal vein passes behind the aorta. This variant, together with the left renal vein around the aortic, constitutes the most frequent anatomical variants of IBV [5].

The presence of the left aortic renal vein can cause clinical symptoms such as abdominal pain and/or hematuria [2] [3]. Our patient came in for abdominal pain that could be explained by this variant.

Anatomical variants of the left renal vein (number, aortic retropath, periaortic venous ring) are of particular importance in renal and vascular surgery. In indications such as segmental nephrectomy, kidney transplant, abdominal aortic aneurysm, retroperitoneal nodule surgery, testicular tumors or porto cave derivations, these variants could influence the operating technique in one way or another [4].

The iliac veins and the bifurcation of the iliac veins derive from the non-regression of the distal portion of the posterior cardinal vein. The variations of the iliac veins result from a poor development of the posterior cardinal system during the separation of the iliac veins. Among these variations, we find: a controlateral drainage of an internal iliac vein as in our observation or more rarely a junction between the two internal iliac veins that form a single trunk before their termination in the VCI [2].

However, these iliac variations are important to recognize in case of pelvic surgery as part of lymph node curage, kidney transplant, or hysterectomy in women. Our patient suffers from benign prostatic hypertrophy and could potentially be treated surgically by urologists.

In some cases, several variants may coexist in the form of a double IVC with a left renal vein retroaortic and azygo and/or hemi azygos prolongation. The association of these three anatomical variants of the inferior vena cava is rare. Ahmatoglu et al. described the same malformations but with different iliac venous variations [2].

The main risk in this patient with Ag HBs is deep vein thrombosis, hence the need for close liver control.

The second main risk is massive hemorrhage during a possible endovascular procedure, biopsy or abdominal or thoracic surgery.

The most commonly used imaging means are phlebography, doppler ultrasound, scanner and MRI. 
Phlebography is the most reliable way but has the disadvantage of being invasive.

Doppler ultrasound is the most practical way, but still limited exploration in case of interposition of digestive gas, or obesity. Because of its cost, the MRI is not a preferred method, although it is non-irradiant.

CT remains the most reliable means, which offers with the latest innovations, images of better "pseudo angiographic» resolution thanks to volume reconstructions.

\section{Conclusions}

A replication of the inferior vena cava associated with retrohepatic agenesis of IBV with azygos continuation was incidentally discovered during an abdominal ultrasound, and was confirmed by CT in a 69-year-old patient. Embryological, anatomical, radiological and clinical aspects were discussed. The diagnosis is based on computed tomography with injection of iodized contrast agent, which reveals these different congenital abnormalities.

Knowledge of these abnormalities is necessary, in order to avoid dramatic surgical complications in case of endovascular or retroperitoneal surgery.

The role of the radiologist is to know how to recognize these different anomalies in order to avoid any misinterpretation.

\section{Conflicts of Interest}

The authors declare no conflicts of interest regarding the publication of this paper.

\section{References}

[1] Mandato, Y., Pecoraro, C. and Gagliardi, G. (2019) Azygos and Hemiazygos Continuation: An Occasional Finding in Emergency Department. Radiology Case Report, 14, 1063-1068. https://doi.org/10.1016/j.radcr.2019.06.003

[2] Ahmetoglu, A. and Cansu, A. (2012) Duplication or the Inferior Vena Cava with Azygos Continuation, Retro Aortique Left Renal and Iliac Vein Variation. Bratislavske Lekarske Listy, 113, 448-450. https://doi.org/10.4149/BLL_2012_100

[3] Coco, D., Cecchini, S. and Leanza, S. (2016) Inferior Vena Cava Duplication: Incidental Case in a Young Woman. Case Reports in Radiology, 2016, Article ID: 3071873. https://doi.org/10.1155/2016/3071873

[4] Chiarugi, M., Fregoli, L. and Iacconil, P. (2015) Inferior Vena Cava Duplication. Updates in Surgery, 67, 325-327. https://doi.org/10.1007/s13304-015-0301-8

[5] Goutet-Leonard, C. (2011) Deep Venous Thrombosis and Abnormality of the Inferior Vena Cava [Thèse d'exercice: Médecine]. Université henry poincaré, Nancy.

[6] Boudier, J. (2016) Interest in Looking for an Abnormality of the Inferior Vena Cava in the Course of a Deep Venous Thrombosis in Children: About a Case of Agenesis of the Inferior Vena Cava and Literature Review [Thesis: Medicine]. University of Training and Research, Rouen.

[7] Bass, J.E., Redwine, M.D., Kramer, L.A., Huynh, P.T. and Harris, J.H. (2000) Spectrum of Congenital Anomalies of the Inferior Vena Cava: Cross-Sectional Imaging 
Findings. RadioGraphics, 20, 639-652.

https://doi.org/10.1148/radiographics.20.3.g00ma09639

[8] Senecail, B., Josseaume, T., Bobeuf, J., Hebert, R., Wazizi, R. and Nonent, M. (2004) Right Duplication of Inferior Vena Cava. Morpho, 88, 183-187.

https://doi.org/10.1016/S1286-0115(04)98146-5

[9] Carboni, F. (2019) Unusual Incidental Finding: Azygos Vein Continuation. Surgery, 165, 1050-1051. https://doi.org/10.1016/j.surg.2019.01.006

[10] Larbi, T., Hamzaoui B'chir, S., Abdallah, M., Harmel, A. and Benmiled, K. (2013) Pseudo-Pott and Azygo Continuation of the Inferior Vena Cava. La revue de médecine interne, 34, A168. https://doi.org/10.1016/j.revmed.2013.03.185

[11] Ito, T. and Ikeda, Y. (2018) A Case of Double Inferior Vena Cava with Renal, Ovarian and Iliac Vein Variation. Anatomical Science International, 93, 139-143. https://doi.org/10.1007/s12565-017-0397-7

[12] Bami, S., Vazquez, Y., Chorny, V., Goldfisher, R. and Amodio, J. (2015) Deep Venous Thrombosis of the Leg, Associated with Agenesis of the Infrarenal Inferior Vena Cava and Hypoplastic Left Kidney (KILT Syndrome) in a 14-Year-Old Child. Case Reports in Pediatrics, 15, e864047. https://doi.org/10.1155/2015/864047

[13] Ismail, E.A., Azab, A.F., Jayappa, S. and Al-Qattan, H. (2010) Congenital Absence of the Infra Hepatic Segment of the Inferior Vena Cava with Deep Venous Thrombosis in an 8.5-Year-Old Boy. Pediatrics International, 52, e117-e120. https://doi.org/10.1111/j.1442-200X.2010.03081.x

[14] Kondo, Y., Koizumi, J., Nishibe, M., Muto, A., Dardik, A. and Nishibe, T. (2009) Deep Venous Thrombosis Caused by Congenital Absence of the Inferior Vena Cava: Report of a Case. Surgery Today, 39, 231-234. https://doi.org/10.1007/s00595-008-3830-7

[15] Coscina, W.F., Arger, P.H., Mintz, M.C. and Goleman, B.G. (1986) Concurrent Duplication and Azygos Continuation of Inferior Vena Cava. The Journal of Computed Tomography, 10, 287-290. https://doi.org/10.1016/0149-936X(86)90057-3

[16] Benayoun, M., Merran, S., Eiss, D. and Helenon, O. (2008) Sectional Imaging of Renal Veins and Inferior Vena Cava: Normal Aspects, Variants and Malformations. EMC, Paris (Elsevier Masson SAS), Radiodiagnostic-Urology-Gynaecology, 34-107A-20. 